\title{
Lyrische Apostrophen zwischen Gedicht und Gebet (Klopstock, Celan)
}

Andreas Mauz

\author{
"Das Du ist der Angeredete schlechthin. \\ Das ist die allgemeine semantische Funktion, \\ und man wird sich fragen müssen, wie die Sinnbewegung \\ der dichterischen Rede diese Funktion ausfüllt." \\ Hans-Georg Gadamer,
}

Wer bin Ich und wer bist Du? (1970)

\begin{abstract}
»Transzendieren des Gedichts ohne Gott und Götter, trotzdem ausgestattet mit dem gehobenen Ton des Sprechens zu einem Du, das als Adressat nicht mehr vorstellbar ist."
\end{abstract}

Günter Kunert,

Der Schlüssel zum Lebenszusammenhang (1984)

\section{I.}

Zur Eigenart lyrischen Sprechens, zur poetischen Lizenz, gehört, dass es anspricht, dass in ihm alles ansprechbar wird, auch das NichtAnsprechbare - sei es eine abstrakte oder mythologische Vorstellung (»O Schicksal«, "O Muse«), ein Toter (»O Sophokles«), ein Abwesender (»O Gleim«), eine Jahreszeit (»Frühling, ja du bist’s!«), der Schlaf (»Heiliger Schlaf?«), eine Stadt (»Du, der Vaterlandsstädte / Ländlichschönste»), ein Körperteil (»Nun geht ihr matten Glieder...») oder ein Gegenstand (wie in Mörikes "Auf eine Lampe« oder Flemings "An seinen Ring«). Der lyrische Text konstituiert sich oft durch die Inszenierung einer Sprechsituation, einer Situation der (An-)Rede. Indem es anspricht, bedarf das lyrische Ich eines - wie immer gearteten - lyrischen Dus. Der lyrischen Personifikation sind, wie die genannten Beispiele andeuten, keine Grenzen gesetzt.

Und gerade dies wirft Fragen auf.Was bedeutet es für die Rede des Gebets, das Wortgebet als wesenhaft adressiertes Reden, wenn das Gedicht sich durch Anrede alles und jedes zu eigen machen kann? Anrede-Lyrik vollführt eine ausgreifende, den Adressaten der Rede in gewisser Weise zurichtende Geste; sie nimmt das Gegenüber hin- 
ein in das eigene Sprechen. Was ist nun über das Verfügungsmoment der Rede zu sagen, wo sie sich auf Gott richtet? Was bedeutet es für das Gebet, wenn Heinz Schlaffer im Bezug auf das Gedicht festhält, dass das apostrophierte $\mathrm{Du}$ "mehr ein Vorwand des präsentischen, bewegten Sprechens als ein Adressat $\aleph^{1}$ sei?

Das Augenmerk gilt im folgenden den Elementen und Operationen, welche die Sprechsituation konstituieren. Ihre Ausprägungen sollen anhand zweier Texte erhoben werden, einer Ode Klopstocks und einem Gedicht Celans. Einige der interpretationsleitenden Fragen seien vorab genannt: Was gibt das Ich durch die Anrede von sich zu erkennen? Welches Verhältnis unterhält es mit der angesprochenen Instanz? Wie lässt sich sein Sprechen charakterisieren? Woraufhin erfolgt die Anrede, was ist ihr Anlass? Wie ist sie situativ und zeitlich verortet? Finden sich Spuren einer Antwort der angesprochenen Instanz? Wird die Anrede zum Dialog? Inwiefern lässt sich das Ich als textinterne Instanz auf das aussertextliche empirische Ich - des Lesers - übertragen? Welcher aneigenbare Rollenentwurf wird erkennbar?

II.

Der Tod

O Anblick der Glanznacht, Sternheere,

Wie erhebt ihr! Wie entzückst du, Anschauung

Der herrlichen Welt! Gott Schöpfer!

Wie erhaben bist du, Gott Schöpfer!

Wie freut sich des Emporschauns zum Sternheer, wer empfindet

Wie gering er, und wer Gott, welch ein Staub er, und wer Gott, Sein Gott ist! O sei dann, Gefühl

Der Entzückung, wenn auch ich sterbe, mit mir!

1 Heinz Schlaffer, Die Aneignung von Gedichten. Grammatisches, rhetorisches und pragmatisches Ich in der Lyrik, in: Poetica 27 (1995), 1-2, 38-57, 43. 
Was erschreckst du denn so, Tod, des Beladenen Schlaf?

O bewölke den Genuss himmlischer Freude nicht mehr!

Ich sink in den Staub, Gottes Saat! was schreckst

Den Unsterblichen du, täuschender Tod?

Mit hinab, o mein Leib, denn zurVerwesung!

In ihr Tal sanken hinab die Gefallnen

Vom Beginn her! mit hinab, o mein Staub,

Zur Heerschar, die entschlief?

Klopstocks Ode $(1764)^{2}$ bietet, betrachtet man sie hinsichtlich ihrer Anredestrukturen, ein recht komplexes Bild. Der Adressat der Anrede ist nicht stabil, vielmehr richtet sie sich gleitend auf eine Vielzahl verschiedener Adressaten, die in spezifischer Reihung aufeinander folgen.

Erster Adressat der Rede des schon zu Beginn reichlich enthusiasmierten Ich ist nicht Gott, sondern, zu ihm vermittelnd, die Sternheere der Glanznacht; als Inbegriff der Hohheit des vollkommenen und ewigen Werks des Schöpfergottes (s.u.) nimmt seine Anrufung den Weg über das Lob der nächtlichen Gestirne, deren Erhabenheit erhebt und zur entzückten Wendung an die gesamte "herrliche Welt" führt (man beachte die alliterierenden "Sternheere", »erhebt«, »herrlichen«, "erhaben«). ${ }^{3}$ Dann erst erfolgt der zweimalige emphatische Anruf des erhabenen Gottes als Schöpfer.

Hier, nach (gleichsam induktivisch) vollzogenem Erkenntnisprozess, der zugleich Hymnus ist, kann in Strophe 2 das Verhältnis von Gott und Mensch abstrakt und verkürzt benannt werden. In kunstvoll-chiastischerVerschränkung (Vers 3) wird die Überlegenheit des Schöpfergottes, wie sie die Betrachtung des "Sternheer" lehrt, in Opposition gebracht zur Hinfälligkeit des Menschen, der mit Gen 2,7 nichts ist als "Staub«. Die Form der Anrede, stets Hinweis auf das Verhältnis von Sprecher und Angesprochenem, stets Beitrag zu einer bestimmten kommunikativen Atmosphäre, impliziert hier deutlich

2 Zitiert nach: Friedrich Gottlieb Klopstock, Ausgewählte Werke, hg. v. Karl August Schleiden, Nachwort v. Friedrich Georg Jünger, München 1962, 101. Für die Geschichte der Gattung kommt Klopstock eine entscheidende Rolle zu. Ihm gelingt die Assimilation der antiken (quantitativen) Metren mit der Rhythmisierung des deutschen Verses nach dem Wortakzent.Vgl. u.a. ders., Von der Nachahmung des griechischen Silbenmasses im Deutschen (1756), in: Klopstock, Ausgewählte Werke, 1038-1048.

3 Das Erhabene ist für Klopstock eine, wenn nicht die zentrale poetologische Kategorie.Vgl.: Das Erhabene in der Dichtung: Klopstock und die Folgen, Städtisches Museum Quedlinburg/Klopstockmuseum (Hg.), Halle 1997. 
Unterordnung und Verehrung. Die schlechthinnige Abhängigkeit des Ichs wird inszeniert durch syntaktische und lautliche Verschlingungen, die Gott und Mensch immer wieder aufeinander beziehen.

Doch gerade weil die Vergänglichkeit des Menschen auf diesem Wege, über ihre Kontrastierung mit dem allmächtigen Sein des sorgenden Gottes, eingeführt wird, ist ihr die anfechtende Spitze von vornherein genommen. So sehr das Vergänglichkeitsbewusstsein auch die Frage nach dem Tod nach sich zieht, wird sie (obwohl in den Strophen 3 und 4 breit ausgestaltet) nie ganz bedrohlich. Noch an der eigenen Vergänglichkeit vermag das Ich sich zu berauschen.

Vom hoffnungsvollen Ruf, auch in der Todesstunde nicht vom Entzückungsgefühl verlassen zu werden motiviert, wird, gleichsam als Probe aufs Exempel, in Strophe 3 eine solche inszeniert. Als neuer (und fünfter) Adressat der Rede betritt in Strophe 3 der feindliche Tod die Bühne. Im Hinblick auf das Gebet als Bitte ist entscheidend, dass sich die Bitte um Bewahrung im Tod hier nicht direkt an Gott richtet, sondern an das eigene "Gefühl der Entzückung« als Inbegriff des Gottesbewusstseins. Die Überwindung des Todes gelingt nicht durch die Präsenz des angerufenen Gottes - sie verdankt sich einer Jenseitsgewissheit, die in der Verzückung über den Sternenhimmel Gestalt besitzt. Das Gebet, als Gebet für den Menschen, richtet sich hier an den Menschen selbst; es ist anthropologisches Beten. Gott ist direkt nur als Schöpfergott angesprochen. Als Garant von Unsterblichkeit, die dem Tod seinen Stachel nimmt, gerät er nur in dieser indirekten Weise in den Blick.

Die metaphorische Anrede des Todes als »Bewölkung" (oder doch: als Bewölkung verursachende Instanz) ist durchaus sinnreich, da der (Todes-)Kampf damit in jener Sphäre vollzogen wird, die dem Ich - wie so emphatisch zum Ausdruck gebracht - als Garant der Allmächtigkeit Gottes gilt. Der »täuschende Tod» verstellt als ängstigende Schwelle den Blick auf die "himmlische Freude«; er stört, bis zur Möglichkeit des Vergessens der eigenen Unsterblichkeit, die Klarsicht auf den Sternenhimmel, der auf den Himmel verweist. Irdischer Himmel und himmlischer Himmel, Todesangst und Jenseitsgewissheit werden hier gleichsam als meteorologisch-theologisches Drama inszeniert, dessen Formel lautet: freie Sicht, Zuversicht.

Durch die Besinnung auf den Wert des Staubs, auf die blosse Schwellenhaftigkeit des Todes, kommt in Strophe 4, als sechster und letzter Adressat der Rede der eigene "Leib» (bzw. wiederum "Staub») in den Blick. Aus der Anfechtung gestärkt hervorgegangen, verfallt das auferstehungsgewisse Ich nun geradezu in eine gesteigerte Affirmation des Todes, zumal dieser breit als Heldentod ausstaffiert wird. 
Retrospektiv betrachtet, erweist sich der Text als eine gekonnte, durch Apostrophen massgeblich bestimmte Verschränkung des Motivfelds Nacht - Schlaf - Tod - Auferstehung. Er nimmt seinen Ausgang von einer nächtlichen Betrachtung des Sternenhimmels, die zum Lob Gottes führt. Dies wiederum zieht die Betonung der eigenen Vergänglichkeit nach sich, die Bedrohung durch den Tod. Die Anfechtung wird als Streit mit dem Tod ausgetragen, der, im Bestehen auf die Klarsicht über den Tod hinaus, zugunsten des Ichs ausgeht. Dass es sich selbst u.a. auch in dritter Person als nächtliche Schlafende (Strophe 3, Vers 1) anspricht, weist voraus auf den Todesschlaf der Gerechten, die der Auferstehung harren. Zu ihnen will das Ich sich nun willig begeben. Die Struktur des Textes folgt einer strengen Logik - des Blicks wie der Anrede -, die von der Schöpfung zu Gott, von dort zum Menschen und seiner Vergänglichkeit führt, die wiederum in Gott aufgehoben ist. Obwohl nicht durchgängig an Gott gerichtet, lässt sich Klopstocks "Der Tod « als (Nacht-)Gebet interpretieren, das Anfechtung mit Hymnus zu verbinden weiss.

Ein letzter Blick zurück auf den Text. Fraglich scheint, ob die Todesszene in den Strophen 3 und 4 als - höchst intensives - Gedankenexperiment wahrzunehmen ist, oder, ungleich dramatischer, als tatsächliche letzte Worte des Ichs. In jedem Fall ist gerade durch die Interjektionen und Apostrophen das (auch in den Prädikaten) Präsentische sehr dominant gestaltet. Sollte die Rede an den Tod als tatsächliche zu verstehen sein, stellt sich allerdings die Frage, wie die lokale Verortung des Sprechers und seine Befindlichkeit vorzustellen sind. Befindet er sich in den ersten beiden Strophen doch zweifelsfrei unter freiem Himmel und bei guter Gesundheit, muss ein plötzlicher Todeskampf irritieren. Aber nicht nur hier fordert der Text eine Rekonstruktion seines Inhalts; durch die starke poetische Gestaltung (Wiederholungen) wirkt etwa auch der zweite Vers der zweiten Strophe vor aller inhaltlichen Bestimmtheit durch seinen Klang.

Was hier vorliegt, ist jene "schöne Unordnung", die Gottsched (Boileau folgend) als Stilprinzip der Ode verstanden wissen will. ${ }^{4}$ Gerade die Verwirrung, die erschwerte Auflösbarkeit des Textes in ein vorstellbares Geschehen, besitzt hier entscheidende Bedeutung. Denn Klopstocks erklärtes Ziel ist nicht eine "descriptive Poesie", eine "poetische Schilderey", wo der Ausdruck »bloss Betrachtung

\footnotetext{
4 Vgl. Johann Christoph Gottsched, Versuch einer critischen Dichtkunst, Leipzig ${ }^{4} 1751,435$.
} 
bleibt, wo er Leidenschaft hätte werden sollen «" , sondern Täuschung durch ein "Herausheben der eigentlichen innersten Beschaffenheit der Sache ${ }^{6}$. Indem der Dichter die emotionalen Potenzen einer Sache - des Sternhimmels etwa - heraushebt, in ihrem Empfindungsgehalt kenntlich macht, stellt er dar. "Darstellung" wird als Gegenbegriff zur "Beschreibung installiert: Ziel ist nicht objektive Wiedergabe, sondern intensivierende Evokation der Wirkung all dessen, was durch die abgestumpfte Wahrnehmung an Emotion und Empfindung verloren geht. ${ }^{7}$

Weltaneignung geschieht nicht in exakter Wahrnehmung und deren Deutung, sondern im Gefühl. Das affektive Gottesbewusstsein wird überhaupt erst erarbeitet und im täuschenden Als-Ob gesteigert dargestellt. Dann allein, wenn »Schlag auf Schlag, Lebendiges Lebendigem folgt", gelingt es, "uns über unsere kurzsichtige Art zu denken [zu] erheben und uns dem Strome [zu] entreissen, mit dem wir fortgezogen werden $\aleph^{8}$.

Das bedeutet allerdings auch: Auf den nächtlichen Gang unter dem Sternenhimmel kann zugunsten der Klopstock-Lektüre im Ohrensessel verzichtet werden. Das ehrfürchtige Schaudern vor dem Sternenhimmel ebenso wie vor dem Tod ist auch durch Lektüre zu gewinnen. Der täuschenden Darstellung der Dinge gelingt, dass sie ganz gegenwärtig und »beynahe die Dinge selbst zu seyn scheinen $"$; mehr als blosse Objektivität zeigt die "schöne Unordnung« den fühlenden Lebensbezug. Eher als von Erlebnislyrik wäre demnach von Erlebnislektüre $^{10} \mathrm{zu}$ sprechen. Das beständige Gleiten des Adressaten der Anrede dient als Moment der Dynamisierung und Steigerung genau diesem Zweck.

"Der Tod« ist ein kathartisches Gebet. Entzücken angesichts des Sternenhimmels und Todesangst werden hier nicht in ein trockenes Bekenntnis zum Auferstehungsglauben übergeleitet, sondern ausgestaltet als interjektionsreiche Rede eines emotional arg gebeutelten,

\footnotetext{
5 Friedrich Gottlieb Klopstock, Gedanken über die Natur der Poesie (1759), in: Ders., Klopstocks sämmtliche Werke, hg. v. August L. Back/Albert R. Spindler, Bd. 16, Leipzig 1830, 33-44, 38.

6 Friedrich Gottlieb Klopstock, Von der Darstellung (1779), in: Ders., Klopstocks sämmtliche Werke, Bd. 16, 2-12, 9.

7 Ebd., 12.

8 Ebd., 7.

9 Ebd., 4.

${ }^{10}$ Vgl. Klaus Weimar, Das Wandeln des Wortlosen in die Sprache des Gedichts, in: Kevin Hilliard/Katrin Kohl (Hg.), Klopstock an der Grenze der Epochen, Berlin 1995, 33-45, 45.
} 
zum Schluss aber nicht nur befriedeten, sondern bis zur Todeslust heroischen Ichs.

Subjektiv ist Klopstocks Darstellung ebenso zweifellos, wie sie nichts von Individualität an sich hat. Das sprechende Ich bleibt hinsichtlich seiner Identität gänzlich unbestimmt, das Dargestellte zielt auf das Vorbildlich-Repräsentative. Es ist sehr viel weniger Darstellung von in einer genannten Situation empfundenen Gefühlen als kalkulierte Evokation derselben beim Rezipienten.

Diese Bemühung macht Klopstock, wie Klaus Weimar gezeigt hat, zum Exponenten eines Projekts, das sich durch die Geschichte der deutschen Lyrik des 18. Jahrhunderts verfolgen lässt und als "Oralisierung der Sprache ${ }^{11}$ bezeichnet werden kann. Spätestens seit der galanten Lyrik Ende des 17. Jahrhunderts etabliert sich der Versuch, "die vermittelnde Schrift vergessen zu machen zugunsten der Illusion unmittelbarer Präsenz von Sprache und Textwelt « ${ }^{12}$. Im Horizont dieses paradoxen Projekts einer schriftlichen Leugnung der Schrift sind Klopstocks Neuerungen innerhalb der poetischen Technik seine "Überwältigungstechniken « ${ }^{13}-\mathrm{zu}$ sehen. Der freie Rhythmus, aber auch bestimmte Manipulationen innerhalb der temporalen Struktur des Textes dienen dazu, »das Lesen zum Hören und Sehen werden zu lassen und also die Differenzen von Schrift, Sprache und Textwelt zum Verschwinden zu bringen $\aleph^{14}$. Unter Textbedingungen muss wiedergewonnen werden, was die Sprache durch die vermittelnde Schrift verliert: Intonation, Mimik, Gestik.

Das Ich, welches Klopstock durch dieses Verfahren dem Leser zur Aneignung anbietet, ist tatsächlich ein vollkommeneres. So wie dieses Ich fühlt sich der wahre Mensch und durch seine Nachhilfe darf auch der Leser dem Tod unerschrocken ins Gesicht sehen - anders als das lyrische Wir des folgenden Textes.

\footnotetext{
${ }^{11}$ Weimar, Das Wandeln des Wortlosen, 33.

12 Ebd.

${ }^{13}$ Ebd., 34.

${ }^{14}$ Ebd., 33.
} 
III.

Psalm

Niemand knetet uns wieder aus Erde und Lehm, niemand bespricht unseren Staub.

Niemand.

Gelobt seist du, Niemand.

Dir zulieb wollen

wir blühn.

Dir

entgegen.

Ein Nichts

waren wir, sind wir, werden

wir bleiben, blühend:

die Nichts-, die

Niemandsrose.

Mit

dem Griffel seelenhell,

dem Staubfaden himmelswüst,

der Krone rot

vom Purpurwort, das wir sangen

über, o über

dem Dorn. ${ }^{15}$

15 Aus: Paul Celan, Die Niemandsrose (1963), in: Ders., Gesammelte Werke in fünf Bänden (= GW), hg. v. Beda Allemann et al., Bd. 1, Frankfurt a.M. 1983, 225. - Der "Psalm» gehört zu den bekanntesten und meist bearbeiteten Gedichten Celans; die Literatur, die das Gedicht hervorgebracht hat, sei es als solches, sei es im Horizont übergeordneter Fragestellungen, ist kaum zu überblicken. Die vorliegende Interpretation verdankt vor allem zwei Arbeiten wichtige Hinweise: Klaus Reichert, Fragendes Verstehen. Zu Paul Celans Gedicht "Psalm«, in: Helmut Brackert/Jörn Stückrath (Hg.), Literaturwissenschaft. Ein Grundkurs, Reinbek 51997, 210-225; Inka Bach/ Helmut Galle, Deutsche Psalmendichtung vom 16. bis zum 20. Jahrhundert. Untersuchungen zur Geschichte einer lyrischen Gattung, Berlin 1989, 378-407. Für theologische Interpretationen u.a.: Achim Müller, Paul Celan, »Psalm«, in: Homiletische Monatshefte 87/5 (2011), 228-231; Jan-Heiner Tück, Gelobt seist Du, Niemand: Paul Celans Dichtung - eine theologische Provokation, Frankfurt a.M. 2000. 
Durch den Titel, »Psalm«, appelliert Celans Text an die Gattungsvorstellung des Lesers; er stellt ihn in eine Tradition, vor der er interpretiert werden kann und will. Psalmistische Rede ist freudige, von Musik begleitete Lobrede, aber auch Klage - Rede auf jeden Fall, in der Israel seinen Gott anspricht, sei es im liturgischen Zusammenhang oder im persönlichen Gebetsleben, sei es als Einzelner oder in Gemeinschaft. Die Rhetorik des Psalms ist eine Rhetorik der Anrede bzw. des Dialogs.

Celans Text knüpft sowohl in Gestus als auch Bildlichkeit vielfach und neu akzentuierend an diese Sprechweise an, ohne dass er sich als Parodie oder Kontrafaktur der Psalmen oder eines bestimmten Psalms beschreiben liesse. Wie einige der alten Psalmen bewegt auch er sich in eigentümlicher Weise zwischen Klage und Hymnus.

Anders als in einer Mehrzahl der Psalmen erfolgt die Anrede hier nicht unmittelbar. ${ }^{16}$ Das Gedicht - der Psalm - setzt vielmehr ein mit einer Reflexion des Sprechersubjekts, einem kollektiven Wir, auf sich selbst, auf seine Verfassung. Niemand knetet es, wieder, aus Erde und Lehm; niemand bespricht seinen Staub. Der in Form eines synthetischen Parallelismus vorgetragene Bezug zur Schaffung des Menschen, wie sie der zweite Schöpfungsbericht in Gen 2,7 überliefert, liegt auf der Hand. Die aus Erde geschaffenen Menschen - Staub, der wieder Staub werden wird - sind, um Leben zu können, angewiesen auf die göttliche Besprechung, auf Beseelung durch den göttlichen Hauch. Sie, die Toten oder doch Versehrten, klagen an, dass sie nicht wieder geschaffen werden können, sie beklagen die Unmöglichkeit einer creatio nova. Niemand kann dies erneut tun.

Niemand kann dies erneut tun. - Erst von der zweiten Zeilengruppe ${ }^{17}$ her erweist sich die Bedeutung des dreimalig genannten Niemand (anaphorisch betont, insbesondere in Vers 3 als Einzelwort; es ist das erste Wort des Textes insgesamt wie erstes und letztes Wort der ersten Zeilengruppe). Durch die lobende Anrufung - hier erfolgt sie nun - entpuppt sich das Niemand als jemand, als jemand, der den Namen Niemand trägt. Was Indefinitpronomen zu sein schien im Sinne eines "Keiner«, der Bezeichnung der Abwesenheit eines »Jemand «, ist - auch - angerufener Name. Die genannte Lesart der ersten Versgruppe muss um eine zweite erweitert werden: Die klagend-anklagende Stimme des Wir verwandelt sich in eine kon-

\footnotetext{
${ }^{16}$ Einschränkend ist festzuhalten, dass eine grosse Zahl der Psalmen Überschriften tragen mit Aussagen zur Autorschaft und situativen Verortung der Rede (u.a. Ps 34, 51, 54 u.ö.). Die Anrede Gottes erfolgt jeweils in Vers 2.

${ }^{17}$ Der ungleichen Verszahlen wegen liegt es nahe, von Zeilengruppen und nicht von Strophen zu sprechen.
} 
statierende, ja bekennende (oder eben: lobende) ${ }^{18}$; das ausschliesslich rückwärtsgewandte "wieder" wird nun lesbar als "jetzt" und "nocheinmal«. Allein: Wer da wieder knetet und bespricht ist und bleibt - Niemand. Der Ort des Schöpfergottes wird besetzt von einem Gott, der Niemand heisst, der vielleicht aber eben doch auch niemand ist. Eröffnet ist damit das "Niemand-Spiel«, wie es sich von der einschlägigen Odyssee-Szene (9. Gesang, Verse 364ff) bis in die Literatur der Gegenwart verfolgen lässt. ${ }^{19}$ Die Negierung Gottes als ein Niemand und die Setzung einer Niemand genannten göttlichen Instanz sind hier zugleich präsent. Im auf Gott bezogenen NiemandSpiel wird Gott angesprochen und - wenn nicht geleugnet, so doch in seinem Sein (Sein zumal für die tote Schöpfung) in Frage gestellt.

Eine eindeutige Leugnung Gottes ist den Versen nicht zu entnehmen, da das Niemand eine Negation besonderer Art ist. Es ist »nicht schlicht leer, nicht reine Abwesenheit, sie ist ein positiv gesetztes Etwas, das einen Namen hat, die Anwesenheit einer Abwesenheit $\mathbb{1}^{20}$. Anrufung und Fraglichkeit der angerufenen Instanz sind so in einer Figur, Kippfigur, zusammengefügt. ${ }^{21}$

Dieser zwiespältige Niemand wird nun gelobt, gelobt auch in Form eines Blühens, dem Niemand entgegen. Die Bewegung, die sich bislang - sei es als be- und eingeklagte, sei es als tatsächlich Geschehende - von oben nach unten, vom Niemand zum Wir vollzog, wird nun von einer lobend-wachsenden Aufwärtsbewegung abgelöst. Stärker akzentuiert (und zugleich textlich inszeniert) wird damit die Opposition und Distanz von Wir und Niemand. (Man beachte das Arrangement Dir - wir - Dir wie auch den oppositionellen Aspekt des »entgegen«, der durch das variierte Enjambement der ansonsten parallel formulierten Verse noch verstärkt wird - "Dir zulieb» und "Dir / entgegen« sind als gegensätzliche Qualifizierungen der einen Bewegung des Blühens zu lesen.) Das vorgetragene Lob ist brüchig,

\footnotetext{
${ }^{18}$ Durch die Struktur der Sätze wird das "Niemand « natürlicherweise gross geschrieben; es bildet eine "grammatikalische Brücke« (Bach/Galle, Deutsche Psalmendichtung, 387) zur zweiten Zeilengruppe. Die einmalige Kleinschreibung in Zeile 2 erweist sich, von ihr her, als orthographisch falsch, stärkt aber gerade darin die oszillierende Bewegung zwischen Nomen und Pronomen.

${ }^{19}$ Vgl. Michael Braun, Untersuchungen zum `Niemand . Beitrag zur Geschichte einer paradoxen literarischen Figur und ihrer Darstellung im Bild, Stuttgart 1994; Hannes Fricke, "Niemand wird lesen, was ich hier schreibe«. Über den Niemand in der Literatur, Göttingen 1998.

${ }^{20}$ Reichert, Fragendes Verstehen, 213.

${ }^{21}$ Man hat die negative Lesart zudem stützen wollen durch den Hinweis auf die Brechung des Schöpfungsbildes durch das unbiblische "kneten«.Vgl. Bach/Galle, Deutsche Psalmendichtung, 380.
} 
verhalten, wie sich, vom Enjambement abgesehen, auch in der Interpunktion (Punkt und nicht Ausrufezeichen) zeigt.

Nach dieser extrovertierten Geste des Wir wendet es sich in der dritten Zeilengruppe wieder ganz sich selbst zu; der ansprechende Bezug auf das Gegenüber entfällt (so auch in der vierten und letzten Zeilengruppe). Er entfällt allerdings nur bedingt, da das Wir sich selbst nun in ganz ähnlicher Weise apostrophiert wie den Niemand: als "Nichts". Als dieses Nichts - rückzubeziehen auch auf die Nichtigkeit des Seins, die in den ersten Zeilen zum Ausdruck kam - besitzt das Wir aber dennoch Sein und zwar in allen drei Dimensionen der Zeit. Es ist, immer, blühend; es ist die Nichtsrose, die Niemandsrose, die dem Gedichtband seinen Titel gibt. Wenn der Anredegestus auch entfällt, ist die Bezogenheit des Wir auf den Niemand inhaltlich doch nach wie vor, ja gesteigert thematisch, da das Niemand (Gottes) und das Nichts (des Wir) sich durchdringen in Gestalt der Nichts- und Niemandsrose, die das Wir ist. Die Intimität der Anrede wird in eine inhaltliche Aussage umgestülpt. ${ }^{22}$

Die Durchdringung zeigt sich nicht allein in der wörtlichen Koppelung beider in der Fügung »die Nichts-, die Niemandsrose«; sie ist auch gegenwärtig im Anspruch des Wir auf ein ewiges Sein. Sein Konjugieren - "waren wir, sind wir, werden / wir bleiben" erinnert an die Offenbarung des Gottesnamens in Ex 3,14; genau so, allerdings im Singular, wäre er aufgrund des hebräischen Verbsystems und seiner Zeitstufen zu übersetzen. ${ }^{23}$

Die aufgebaute Opposition wird relativiert, blühend verbindet sie das Nichts mit dem Niemand. Die eine Rose trägt zwei Namen: Nichtsrose, Niemandsrose. Sie ist die Rose des Nichts, die, substanziell, vom Niemand stammt, »Rose des Niemand « aber auch die »Rose für Niemand«. Akzentuiert man den Doppelpunkt in Zeile 12, erweist sich "die Nichts-, die / Niemandsrose« als Zusammenfassung des zuvor Gesagten: Die beschriebene Beziehung wird in die Metapher der Rose erhoben. Sie verdichtet die zentralen Aussagen - Wir waren/sind/werden bleiben ein Nichts. Wir loben Niemand« - zu einem Wort.

Die vierte Zeilengruppe unterzieht nun die Rose einer unerwarteten Diagnose. Sie ist nicht Gegenstand ästhetischer Betrachtung und symbolischer Befrachtung; sie wird zerlegt in Griffel, Staubfaden, Krone und Dorn - gleichsam um Rechenschaft abzulegen, womit sie denn blühe (das Verb »blühen« kann von der dritten auf

\footnotetext{
${ }^{22}$ Es zeigt sich, dass das Niemand in den drei Zeilengruppen eine Bewegung vom "Subjekt« zum vokativisch adressierten Objekt, zum Kompositum beschreibt.

${ }^{23}$ Ich folge hier der Deutung Reicherts, Fragendes Verstehen, 216.
} 
die vierte Zeilengruppe, der ein Prädikat fehlt, übertragen werden). Die botanische Begrifflichkeit ist ihrerseits wiederum mehrfach besetzt. Einerseits werden, anknüpfend an die erste Zeilengruppe, die Schöpfungs- bzw.Vergänglichkeitsthematik, jene Teile der Blüte benannt, die für die Fortpflanzung notwendig sind (wobei Staubbeutel und Fruchtblatt ungenannt bleiben und die Rose, analog zur ersten Lesart der ersten Zeilengruppe, vielleicht ohne Möglichkeit der Fortpflanzung/Neuschöpfung bleibt). ${ }^{24}$ Andererseits markiert die Reihung Krone - rot - Purpur(wort) - Dorn zugleich ein christologisches Feld. Purpur(rot) ist nicht nur die Rose (die Königin der Blumen), es ist auch die Farbe der Könige, des Mantels, der dem dornengekrönten »König der Juden« übergelegt wird (Mk 15,17 par; Joh 19,5), der am Kreuz sein Blut vergiesst. Die Menschwerdung Gottes - Jesus als der fleischgewordene Logos (Joh 1,14) - und die Passion Jesu werden in dem einen "Purpurwort» enggefuihrt. ${ }^{25}$

Über dem assoziativen Hof der Einzelwörter darf aber deren präzise Anordnung und Bezogenheit nicht vernachlässigt werden. Die Substantive sind, exakt parallel formuliert, begleitet durch adjektivische Bestimmungen. Eigenart dieser Epitheta ist aber gerade, dass sie die botanische Konkretheit konterkarieren; sie entziehen die Niemandsrose der sinnlichen Erfahr- und Vorstellbarkeit. Reale und irreal-neologistische Beschreibung, Konkretion und Abstraktion entfalten das Paradoxon der "Niemandsrose", ihre gefährdete Existenz aus und zu Niemand hin. ${ }^{26}$ Sie auf das Substantiv, das sie näher bezeichnen, hin zu interpretieren, fällt schwer. Bezieht man sie aber aufeinander, zeigen sich Korrespondenzen. Auch in ihnen sind die vertikale Stufung und die Schöpfung präsent. Deutlich wird dieser Zusammenhang zumal in der oxymoronhaften Fügung von Genesis-Vokabular (Gen 1,1) zum Neologismus "himmelswüst« - zu lesen hin auch auf den Aufenthaltsort des Niemand. Die Helle, Schöpfungshelle?, die man gängigerweise dem Himmel zuschreibt, gilt hier der Seele, wie sie, im Gegensatz zum Himmel, auch hell, Hölle, ist.

Schliesslich - und das ist für die hier angezielte Interpretation entscheidend - birgt die letzte Zeilengruppe ein deutlich selbstreferentielles Moment. Der Griffel ist nicht nur Teil des Zeugungsappa-

24 Wiederum ein Hinweis Reicherts, vgl. Fragendes Verstehen, 218.

${ }^{25}$ Wie zentral man die christologische Lesart setzen will, ist offen; Celan selbst hat davor gewarnt, den "Psalm» nur von hier zu lesen (vgl. Bernhard Böschenstein, Gespräche und Gänge mit Paul Celan, in: Bernhard Böschenstein/Giuseppe Bevilacqua, Paul Celan. Zwei Reden, Marbach 1990, 7-19, 8). Eindeutig ist aber: Der Christus, der hier in den Blick kommt, ist der Leidende, der vere homo, der Jude Jesus.

${ }^{26}$ Vgl. Bach/Galle, Deutsche Psalmendichtung, 399. 
rates der Rose; er ist auch Schreibwerkzeug des Dichters, das, analog zum Griffel der Rose, die Niemandsrose - Titel des Gedichtbandes, dem der »Psalm» entstammt - hervorbringt. Das »Purpurwort, das wir sangen " ist das Gedicht, zunächst das Gedicht "Psalm", das musikalisch psalmodierend ausklingt auf den Lobe-Vokal »o«, dann aber auch die Gedichte des ganzen Bandes. Auch sie sind »über, o über / dem Dorn", dem Stachel des anfechtenden Todes, gesungen.

Nicht bedacht wurde bislang das "Wir» des Gedichts. Auch hier vermag die Deutung nicht mehr als Hinweise zu geben aufVerständnismöglichkeiten. "Wir«, das sind, betont man den Schöpfungsaspekt, zunächst "wir Menschen (als Tote)«; "wir«, das sind konkreter aber auch "wir Juden (als Ermordete)«, wenn man die (un-)mögliche Neuschöpfung betont durch einen Niemands-Gott, der fraglich, der nur abwesend anwesend ist. "Staub« kann bei Celan kaum gelesen werden ohne Gedanke an die Shoa. Das "Wir« vor allem als jüdisches "Wir« zu verstehen, legen sowohl der Titel nahe (der Psalm als die historische jüdische Form der Klage) als auch die angesprochenen christologischen Implikationen: Jesus, der leidende Jude, der nach Mk und Mt am Kreuz ein Psalmwort, Ps 22,2, betet, wird hier, als Individuum, übertragen auf das kollektive jüdische Wir.

Celans "Psalm« als Anti-Psalm zu bezeichnen wäre falsch. Als Gefäss einer Sprache, die sich an Gott wendet, als spezifische Sprachgeste, bleibt er bestehen. Allerdings vollzieht sich die Rede in einer Weise, die kenntlich macht, wie erschüttert ihre überlieferten Koordinaten sind. Celans "Psalm « ist ein Psalm - ein Psalm aber, der, wie Celan in seiner Büchner-Rede schreibt, »seiner Daten eingedenk « ${ }^{27}$ bleibt. Und das heisst: Der lobend und klagend Angesprochene ist nicht mehr der "Herr der Heerscharen" (Ps 80,20), der "grosse Gott" (Ps 70,5), der »eine feste Burg" (Ps 46,1) ist, er ist - Niemand. (Und vielleicht ist Niemand, wie Odysseus, gerade dadurch gerettet, dass er Niemand geworden ist.)

Dieser Niemand aber ist jemand in dem Sinne, dass er durch die Rede Kontur gewinnt. Er ist jemand, der auf ein Thema, das der (Neu-)Schöpfung, behaftet wird. Es bleibt nicht bei der blossen Ausrichtung, der prädikationslosen Apostrophe an ein gänzlich unbestimmtes Gegenüber. ${ }^{28}$ Die angeredete Instanz, obwohl Niemand,

${ }^{27}$ GW, Bd. III, 196. Büchners Lenz geht am »20. Jänner [...] durchs Gebirg«; Celans 20. Jänner ist der 20. Januar 1942, das Datum der Wannsee-Konferenz, welche die "Endlösung der Judenfrage« beschliesst.

${ }^{28}$ Das ist - vom Psalm her - gegen die Auslegung Derridas und, Derrida folgend, Hamachers festzuhalten.Vgl. Jacques Derrida, Schibboleth. Für Paul Celan (1986), Wien 1986, 91 u.ö.; Werner Hamacher, Bogengebete, in: Norbert Haas, et al. (Hg.), Aufmerksamkeit, Eggingen 1998, 11-44. 
besitzt Kontur; das kollektive Wir, das Nichts, die Niemandsrose versteht sich in seiner Existenz von diesem Niemand her. Ihm gilt das gezeichnete Purpurwort des jüdischen »Psalm».

Der klagende Ruf nach Neuschöpfung macht Celans Text als Bittgebet lesbar. Durch die Art, wie die Bitte vorgebracht wird und die gänzlich offene Besetzung der Position des Angeredeten entfallen hier aber jene Probleme, die es bezüglich der Gotteslehre üblicherweise mit sich bringt. ${ }^{29}$ Wenn die Bitte denn Bitte ist und nicht sachliches Konstatieren einer im Moment geschehenden Neuknetung, kollidiert sie dennoch in keiner Weise mit den Eigenschaften des Niemand. Wie dürfte man Niemand bitten? Wie dürfte man von Niemand Neuschöpfung erwarten?

\section{IV.}

Hier muss schliesslich auf die oben exponierte Frage eingegangen werden:Was ist über das Verfügungsmoment zu sagen, das die Anlage der Rede als Anrede zwangsläufig mit sich bringt? - Es ist in Klopstocks Text zweifellos stärker ausgebildet als in dem Celans. Aller Unterordnung zum Trotz schreibt Klopstocks Ich seine Gegenüber fest. Kenntlich wird dies zunächst in der grossen Spannung zwischen einem Schöpfergott, dessen den Tod überwindende Macht die nächtliche Betrachtung des Himmels lehrt, und einem Niemand, der mehr Indikator einer Abwesenheit, Markierung einer Leerstelle ist. Es zeigt sich darüber hinaus aber auch in den hinsichtlich der Rezeption gegensätzlich angelegten Textstrategien. Anders als Klopstock, der sich um eine Oralisierung des Texts bemüht, um die maximierte Suggestion mündlicher Rede, ist die Rede des "Psalms", was sie ist, wesentlich durch ihre Schriftlichkeit, indem etwa parallel strukturierte Sätze durch Abweichungen im Enjambement weiter semantisiert werden oder durch die Nuancierung, welche eine der inhaltlichen Aussage gegenläufige Interpunktion bedeutet. Die explizite Schriftlichkeit des "Psalm» korrespondiert mit seinem zur Aneignung wenig geeigneten lyrischen Wir ebenso wie mit dem fraglichen Status der angesprochenen Instanz. Im Gegenzug korrespondiert Klopstocks Gestaltung hin auf Mündlichkeit mit dem ohne weiteres aneigenbaren Ich und der Intaktheit Gottes.

Damit sind, als grober Kontrast gezeichnet, zwei Ausprägungen der - man könnte sagen - Physiognomie des lyrischen Du benannt.

\footnotetext{
${ }^{29}$ Vgl. etwa: Wilfried Härle, Den Mantel weit ausbreiten. Theologische Überlegungen zum Gebet, in: NZSTh 33 (1991), 231-247.
} 
Ihnen sind abschliessend und unter Absehung von detaillierten Analysen zwei andere an die Seite zu stellen, welche die vorzutragende These weiter stützen sollen.

Stärker als zu sagen "Gott ist tot" ist zu sagen "Du, Gott, bist tot". Weil Art und Inhalt der Anrede den Angeredeten qualifizieren, kann sie auch in Zeiten geschwundener Gewissheit um die Existenz und Eigenart des Adressaten nicht entfallen. Im Gegenteil: Der Grad seines Verschwindens und die damit verbundene Gefühlslage des Ichs - sei sie die eines willkommenen Abschieds oder die nostalgischer Erinnerung an Zeiten, da man noch beten konnte - ist gerade in dieser Form am präzisesten darstellbar. Die eigentümliche Dialektik der Anrede des toten Gottes liegt darin, dass sie ihm in der Anrede Existenz verleiht, um sie ihm dann durch den Inhalt der Anrede differenziert abzusprechen. Die Funktion der Anrede ist damit eine neue: Sie lässt bewusst Aussage und Aussageform kollidieren; sie spricht nicht um mitzuteilen, sie spricht, um die Unmöglichkeit der Mitteilung darzustellen, sie gestaltet ihre eigene Fragwürdigkeit.

In der Anrede kann Gott mitgeteilt werden, dass man nicht mehr mit ihm rechnet, sei es, wie in Christine Lavants (1915-1973) Theodizee-Text "Gesteinigt hänge ich am Lebensrad « ${ }^{30}$, weil er nicht hinhört, sei es, wie in Miguel de Unamunos (1864-1936) »La oración del ateo $\aleph^{31}$, weil es ihn nicht gibt. In beiden Fällen liegt der tragische Reiz in der offengelegten Fragwürdigkeit der Anrede.

Einen Verdacht weckt derartige Rede allein durch ihre Anlage allerdings unweigerlich: dass in ihr ein Rest von Erwartung mitschwingt, dass sie doch nicht ins Leere spreche, dass da trotz allem ein Adressat sei, der hinhört. Und dies vielleicht auch deshalb,

\footnotetext{
30 "[...] Mein Herz pocht trotzdem solche Morsezeichen, / wie nur die letzte Angst sie einen lehrt. / Und noch im Fallen fühl' ich unversehrt: / sie werden niemals dein Gehör erreichen." Christine Lavant, Kunst wie meine ist nur verstümmeltes Leben. Nachgelassene und verstreut veröffentlichte Gedichte, Prosa, Briefe, ausgewählt u. herausgegeben v. Armin Wigotschnig/Johann Strutz, Salzburg 1978, 86.

31 „GEBET DES ATHEISTEN // Hör meine Bitte, DU GOTT, der DU nicht bist / und in deinem Nichts bist - hör meine Klagen, / DU, der DU die armen Menschen niemals lässt / ohne falsche Tröstung. Widersetz dich nicht / unsern Bitten und unserm Verlangen - DU kennst es. / Und mehr DU von meinem Geist dich entfernst, / mehr erinnere ich die sanften Märchen meiner Seele, / die traurige Nächte mir versüssten. / Wie gross bist DU, mein GOTT! So gross bist DU, / dass DU nichts bist als IDEE; eng ist sie / die Realität, so sehr sie sich auch dehnt, / dich zu umschliessen. Auf deine Kosten leide ich, / GOTT, der DU nicht bist, gäb es dich / gäb es mich - auch mich, ganz ehrlich." Miguel de Unamuno, nach: Vicente Gaos, (Hg.), Diez siglos de poesía castellana, Madrid 1975, 336 (meine Übersetzung).
} 
weil gerade die ins Gesicht gesagte Lästerung als »Köder «2 ${ }^{32}$ wirken könnte - um ein in dem Zusammenhang verwendetes Wort Lavants aufnehmen -, der ihn den Gegenbeweis antreten lässt.

Die paradoxe Anrede des Nicht-Existenten, Nicht-Hörenden, reisst den Adressaten, ob sie will oder nicht, aus seiner Nicht-Existenz, seinem Nicht-Hören, heraus, mindestens für die Dauer der Anrede. Die Rede, die betont, dass sie ungehört verhallen wird (und damit vielleicht auch ein bisschen kokettiert) - „Empfänger unbekannt Retour à l'expéditeur « ${ }^{33}$-, weiss, dass zumindest der Leser sie hört.

- Dr. des. Andreas Mauz ist Oberassistent am Lehrstuhl für Systematische Theologie, insb. Hermeneutik und Fundamentaltheologie und arbeitet an einem Habilitationsprojekt zur interdisziplinären Wissenschaftsgeschichte von evangelischer Theologie und deutscher Literaturwissenschaft.

${ }^{32}$ Vgl. "Jesus Christus, ich bete und bete ...«, in: Christine Lavant, Die Bettlerschale, Salzburg 1956, 145.

${ }_{33}$ Dies der Titel eines Dank-Gedichts/Gebets Hans Magnus Enzensbergers: Vielen Dank für die Wolken. / Vielen Dank für das Wohltemperierte Klavier / und, warum nicht, für die warmen Winterstiefel. / Vielen Dank für mein sonderbares Gehirn / und für allerhand andere verborgene Organe, / für die Luft, und natürlich für den Bordeaux. [...] «, Hans Magnus, Enzensberger, Kiosk. Neue Gedichte, Frankfurt a.M. $1995,124$. 